\title{
The influence of the toxic effect of zinc and mineral starvation on the growth and development of buckwheat regenerants in vitro culture
}

\author{
Borovaya S.A.*, Klykov A.G. \\ Federal Scientific Center of Agricultural Biotechnology in the Far East named after A.K. Chaika, \\ Ussuriysk, Russia \\ * email: borovayasveta@mail.ru
}

Biotechnological methods in breeding are effective in obtaining lines of cultivated plants with improved economically valuable traits and creating completely new genotypes on their basis. Cell-tissue selection, based on the selection of samples that are resistant to a stress factor, take a significant place in solving these problems. The use of heavy metal ions in vitro as selective backgrounds can significantly expand the genetic basis and lead to the emergence common buckwheat forms with new traits and a high potential for resistance to abiotic stressors. The purpose of this work was to study the morphometric traits and adaptive potential of buckwheat regenerant plants of Izumrud and Dikul varieties in vitro culture, resistant to the toxic effect of zinc ions and mineral starvation. The genotypes of common buckwheat: the determinant variety Dikul, selected by the All-Russian Research Institute of Leguminous Crops, and the indeterminate variety Izumrud, selected in the "FSC of Agricultural Biotechnology in the Far East named after A.K. Chaika" were used as source material. The culture was injected with mature seeds, sterilized with concentrated sulfuric acid according to the method of V.A. Tilba, which were then passaged on a hormone-free Murashige and Skoog culture medium (hereinafter MS). To create selective conditions, zinc salt $\left(\mathrm{ZnSO}_{4} \times 7 \mathrm{H}_{2} \mathrm{O}\right)$ was used at concentrations of $808,909,1010,1111,1212$, and $1313 \mathrm{mg} / \mathrm{l}$ in the MS nutrient medium containing $20 \mathrm{~g} / \mathrm{l}$ of sucrose and $6 \mathrm{~g} / \mathrm{l}$ of agar. To simulate mineral starvation, the MC nutrient medium without the addition of macrosalts was used. Induction of direct regeneration of the studied buckwheat genotypes on selective media showed their high tolerance to ionic stress caused by lethal and sublethal doses of $\mathrm{ZnSO}_{4}$. According to the results of 33-day cultivation of test tube microscopes on media with zinc toxicity, 73.8$75.0 \%$ of plants were selected. Secondary cultivation of regenerants under conditions of complete mineral starvation in vitro turned out to be the strongest inhibiting factor for buckwheat. At the same time, a higher level of stress resistance and regeneration capacity was found in the Dikul variety, the morphometric parameters of which were significantly higher than in the Izumrud variety. As a result of the research, the most resistant to the complex stressor buckwheat genotypes were identified. 